\title{
Relevancia de la menopausia y del apoyo social en las diferencias en salud entre mujeres y hombres*
}

\section{Relevance of the Menopause and Social Support in Gender} Differences in Health

Recepción: 21 Junio 2016 | Aprobación: 23 Enero 2017

\author{
Ma Pilar Matud Aznar \\ Universidad de La Laguna, España \\ ORCID: http://orcid.org/0000-0001-5260-1092 \\ Ma Concepción Correa Reverón \\ Universidad de La Laguna, España \\ Livia García PÉrez \\ Universidad de La Laguna, España
}

a Autor de Correspondencia. Correo electrónico: pmatud@ull.es

Para citar este artículo: Matud Aznar, M.P., Correa Reverón, M.C., \& García Pérez, L. (2017). Relevancia de la menopausia y del apoyo social en las diferencias en salud entre mujeres y hombres, Universitas Psychologica,16(1), 1-11. http://dx.doi.org/10.11144/J averiana.upsy16-1.rmas

\begin{abstract}
RESUMEN
Se analizó la relevancia de la menopausia y el apoyo social en las diferencias en salud entre mujeres y hombres mediante un estudio transversal realizado con 710 mujeres y 606 hombres de la población general española con edades entre 20 y 65 años. Se encontró que, aunque en la juventud apenas había diferencias entre hombres y mujeres, a partir de la perimenopausia las mujeres informaban de más insomnio y síntomas vasomotores que los hombres, así como de más dolor durante la postmenopausia. El apoyo social percibido se asociaba con mejor salud, sobre todo en las mujeres en postmenopausia y premenopausia.

Palabras clave

menopausia; género; salud; síntomas somáticos; apoyo social.
\end{abstract}

\begin{abstract}
This study examined the relevance of the menopause and social support in gender differences in health. A cross-sectional survey of a general population sample comprising 710 women and 606 men, aged between 20 and 65 was carried out. No health differences were found between younger men and women. However, perimenopausal women reported more insomnia and vasomotor symptoms, as well as they reported more pain than men through the postmenopause. Perceived social support was associated with better health, especially in premenopausal and postmenopausal women.
\end{abstract}

Keywords

menopause; gender; health; somatic symptoms; social support.

\section{Introducción}

Tradicionalmente se ha considerado que las mujeres tienen peor salud que los hombres, pero los estudios realizados en las últimas décadas han retado la existencia de una desventaja generalizada de las mujeres en cuanto a salud (Walters, 
McDonough, \& Strohschein, 2002), pues han mostrado la complejidad de tales diferencias y han planteado la necesidad de reevaluar las asociaciones entre el género y la salud (McDonough \& Walters, 2001). Se ha destacado la relevancia que en tales diferencias pueden tener factores tales como la edad, los recursos materiales y personales, el apoyo social y el tipo de trabajo. Sin embargo, aunque algunas diferencias en salud entre mujeres y hombres, tales como la morbilidad psiquiátrica menor, no se detectan cuando el estudio se realiza con personas del mismo nivel laboral, se mantiene la mayor prevalencia de síntomas físicos en las mujeres respecto a los hombres (Emslie, Hunt, \& Macintyre, 1999). La mayor presencia de síntomas físicos en mujeres que en hombres con igual ocupación también se ha detectado en estudios realizados en España y en países latinoamericanos (cfrs. Herrera \& Guarino, 2008; Extremera, Rey, \& Pena, 2010).

Existe evidencia de que las mujeres informan con mayor frecuencia que los hombres de síntomas físicos tales como dolores de espalda, de cabeza, palpitaciones, insomnio, o fatiga, entre otros (Herrera \& Guarino, 2008; Kroenke \& Spitzer, 1998; Rincón \& Guarino, 2008; SaizGonzález, Rodríguez, García, Prieto, \& Saiz-Ruiz, 2009; Sánchez \& Ledesma, 2009; Walters et al., 2002). Tales diferencias se han encontrado tanto en muestras clínicas como en población general. Y es más común que dicha sintomatología se asocie con el estrés en las mujeres que en los hombres (Extremera et al., 2010; Moral-de la Rubia et al., 2010).

Las diferencias de la salud entre mujeres y hombres se han explicado tradicionalmente por las diferencias biológicas asociadas al sexo, especialmente por la fisiología endocrina femenina y, tanto a nivel científico como popular, se ha considerado la menopausia como un factor central en los problemas de salud de las mujeres a partir de la mediana edad. La menopausia marca el final de la fase reproductiva de la vida de la mujer y actualmente se reconoce que coincide con un rango de síntomas que se experimentan durante la madurez (Mishra $\&$ Kuh, 2012). Tal y como plantea un grupo científico de la Organización Mundial de la Salud (OMS) de Investigaciones sobre la Menopausia, el cese de la función ovárica que se da en la menopausia hace que disminuya la producción de esteroides y péptidos hormonales produciéndose en el organismo diversos cambios fisiológicos, algunos de los cuales son "resultantes del cese de la función ovárica y de fenómenos menopáusicos con ella relacionados, y otros debidos al proceso de envejecimiento" (OMS, 1981, p. 1).

Aunque, de forma estricta, la menopausia se define como el cese definitivo de la menstruación, en términos populares y de sociedades internacionales para su estudio, generalmente se utiliza para referirse a los años próximos al último periodo menstrual (Sturdee \& Villiers, 2011). En torno a dicho proceso se ha establecido una serie de periodos que se definen a continuación, siguiendo la conceptualización de la OMS (1996). La menopausia natural se define como la desaparición definitiva de la menstruación como consecuencia de la pérdida de la actividad folicular ovárica y se considera que tiene lugar después de un periodo de 12 meses consecutivos de amenorrea, para la que no existe otra causa patológica o fisiológica. La perimenopausia representa el periodo de tiempo anterior a la última menstruación y se caracteriza por un aumento en la variabilidad de los ciclos menstruales; el término premenopausia se recomienda que se utilice para referirse a todo el periodo reproductor hasta la última menstruación; y postmenopausia se refiere al periodo temporal tras la última menstruación, independientemente de si la menopausia es inducida o espontánea. Según la OMS, en todo el mundo, la menopausia natural se produce entre los 45 y 55 años y en las sociedades industrializadas el promedio de edad es de unos 51 años.

Son muchos los estudios que han pretendido identificar y clasificar los síntomas experimentados por las mujeres durante la menopausia, pero no hay unanimidad al respeto. Además, se ha encontrado variabilidad en la frecuencia y severidad de los síntomas en las distintas fases (Duffy, Iversen, \& Hannaford, 2013; Pimenta, Leal, Maroco, \& Ramos, 2012; 
Woods \& Mitchell, 2005), también varían según la cultura y grupo étnico (Avis et al., 2001; Dhanoya et al., 2016; Romero-Pérez, Monterrosa-Castro, \& Paternina-Caicedo, 2010) y en función de variables sociodemográficas, psicosociales y de estilo de vida (cfrs. Dhanoya et al., 2016; Duffy et al., 2013; Cuadros et al., 2012), así como del apoyo social (MartínezGarduño, González-Arratia, van Barneveld, \& Domínguez-Espinosa, 2012). Los síntomas físicos más asociados con la menopausia son los vasomotores (sofocos y sudores nocturnos) y urogenitales, aunque también se han citado otros de tipo cardiovascular, dolores de cabeza, osteoarticulares e insomnio (Avis, Brockwell, \& Colvin, 2005; García-Astudillo, Pinto-García, \& Laguna-Sáez, 2015; Martínez-Pérez, Chavida, Palacios, \& Pérez, 2008; Słopień et al., 2015; Woods \& Mitchell, 2005).

Actualmente se reconoce que muchos de los síntomas asociados tradicionalmente con la menopausia son independientes del género, pues existe evidencia de que también los hombres pueden experimentar muchos de ellos (Calvaresi \& Bryan, 2003; Van Hall, Verdel, \& Van der Velden, 2009; Spetz, Fredriksson, \& Hammar, 2003; Woods \& Mitchell, 2005); pese a ello, se siguen manteniendo una serie de creencias y estereotipos negativos respecto a la menopausia, la cual se asocia con una serie de problemas de salud de las mujeres (cfrs. Melián, 2015; Mendoza et al., 2013; Marcus-Newhall, Thompson, \& Thomas, 2001). Dada la carencia de estudios en los que se tiene en cuenta la relevancia de la edad, así como la comparación entre mujeres y hombres con un mismo nivel educativo, se consideró pertinente realizar este estudio cuyos objetivos son: 1) Estudiar las diferencias en sintomatología entre mujeres y hombres a lo largo de la fase adulta, analizando la relevancia que el estatus menopáusico tiene en dichas diferencias. 2) Analizar la relevancia que el apoyo social tiene en la salud de mujeres y hombres en la adultez.

\section{Método}

\section{Participantes}

La muestra está formada por un total de 1316 personas, de las que el $46 \%(n=606)$ son hombres y el $54 \%(n=710)$ mujeres. El rango de edad es entre 20 y 65 años, si bien no se incluyeron personas con edades entre $32 \mathrm{y}$ 40 años. La media de edad de los hombres es de $44.14(D T=11.65)$ y de las mujeres de $44.11(D T=10.62)$, diferencias que no son estadísticamente significativas, $t(1314)=-0.05$, $p=0.96$. Su estado civil es diverso, aunque la mayor parte de las personas participantes (el 74.6 \%) estaban casadas. El $13.4 \%$ están solteras, casi la décima parte divorciadas o separadas y es mucho menos frecuente que hayan enviudado (el $2.8 \%$ ). Su número de hijos oscila entre 0 y 8 , siendo la media de 1.93 , la desviación típica de 1.42 y la mediana y la moda de 2 . Lo más común es que hayan cursado estudios básicos (42.2\%), si bien más de la mitad ha realizado estudios medios (el 28.2\%) o universitarios (el 29.6 $\%)$, porcentajes que no diferían entre mujeres y hombres, $\chi^{2}(2,1170)=5.30, p>0.05$. La profesión que desempeñan las personas que componen la muestra es variada. El $19.8 \%$ eran mujeres que no trabajaban fuera del hogar y se autodefinían como "amas de casa". Casi la tercera parte (el $30.9 \%$ ) tenía una profesión de tipo manual, el $23.5 \%$ desempeñaba un trabajo de tipo no manual y prácticamente la cuarta parte (el $25.7 \%$ ) eran profesionales para cuyo desempeño es imprescindible haber finalizado una carrera universitaria, de diplomatura o de licenciatura.

\section{Instrumentos}

Inventario SF de Síntomas Físicos. Inventario elaborado a partir de The General Health Checklist (HSQ) (Meadows, 1989). En su versión inglesa está formado por 28 ítems que miden las quejas físicas comunes más frecuentes hechas a los médicos generales y dos ítems 
adicionales solo para mujeres. Estos ítems fueron ampliados de forma que permitiesen conocer el estatus menopáusico de las mujeres, tal y como es definido por la OMS (1996). De los ítems de este inventario se utilizaron los que recogen la sintomatología citada generalmente como asociada a la menopausia, realizando la siguiente agrupación: 1) síntomas vasomotores, que incluye sofocos, o sensación de calor y escalofríos; 2) algias, formada por cuatro ítems: dolores articulares, dolores de cabeza/migraña, dolores musculares, dolor de espalda y dolor de cuello; 3) hipertensión; 4) palpitaciones; 5) insomnio.

Inventario de Apoyo Social (AS, Matud, 1998 ). Instrumento diseñado para la evaluación funcional y global del apoyo social percibido. Está formada por 12 ítems que muestrean las dimensiones de estima, información, compañía y ayuda tangible del apoyo social y se estructura en dos factores: apoyo emocional, formado por siete ítems cuya consistencia interna es de 0.84 ; apoyo instrumental, formado por 5 ítems con una consistencia interna de 0.78 (Matud, Ibáñez, Bethencourt, Marrero, \& Carballeira, 2003).

\section{Diseño}

Se trata de un estudio transversal con cuatro grupos de mujeres y otros cuatro de hombres cuyas edades son similares a las de las mujeres, tanto en rango como en edad media y en desviación típica (véase Tabla 1). Las mujeres mayores de 40 años han sido agrupadas en tres categorías en función de su estatus menopáusico, tal y como lo define la OMS (1996): 1) Premenopausia. Formado por 253 mujeres con edades comprendidas entre 41 y 51 años que no presentaban problemas menstruales ni amenorrea. 2) Perimenopausia. Formado por 83 mujeres que se encontraban en un periodo anterior a la última menstruación y, si bien no tenían amenorrea permanente, presentaban variabilidad en su ciclo menstrual. 3) Postmenopausia. Formado por 129 mujeres con menopausia natural. Además, se incluyó otro grupo de 155 mujeres con edades entre 20 y 31 años, ninguna de las cuales tenía menopausia. Los hombres se dividieron en cuatro grupos en función de su edad de modo que sus edades correspondiesen con las de los cuatro grupos de mujeres. Así, el quinto grupo del estudio incluyó a 155 hombres cuyas edades estaban entre 20 y 31 años; el sexto grupo lo integraba un total de 240 hombres cuyas edades estaban entre 41 y 51 años, que era el rango de edad de las mujeres en premenopausia; el séptimo se formó con 83 hombres cuyo rango de edad era de 44 a 56 , el mismo de las mujeres en perimenopausia; y el octavo incluyó un total de 125 hombres cuyas edades se situaban entre 52 y 65 años, que correspondía al rango de edad del grupo de mujeres en postmenopausia.

\section{TABLA 1}

Edad de los diferentes grupos que componen la

\begin{tabular}{|c|c|c|c|c|}
\hline Grupo & Rango & $M$ & DT & $\mathrm{N}$ \\
\hline 1. Mujeres con edad entre 20 y 31 años & $20-31$ & 27.34 & 3.13 & 155 \\
\hline 2. Mujeres en premenopausia & $41-51$ & 45.21 & 2.59 & 253 \\
\hline 3. Mujeres en perimenopausia & $44-56$ & 50.19 & 2.41 & 83 \\
\hline 4. Mujeres en postmenopausia & $52-65$ & 58.75 & 3.85 & 129 \\
\hline 5. Hombres con edad entre 20 y 31 años & $20-31$ & 26.67 & 3.12 & 155 \\
\hline 6. Hombres con edad entre 41 y 51 años & $41-51$ & 45.20 & 2.75 & 211 \\
\hline 7. Hombres con edad entre 44 y 56 años & $44-56$ & 50.40 & 2.69 & 83 \\
\hline 8. Hombres con edad entre 52 y 65 años & $52-65$ & 57.95 & 3.92 & 125 \\
\hline
\end{tabular}

Fuente: elaboración propia.

\section{Procedimiento}

Todas las personas que participaron en la investigación fueron voluntarias y no recibieron compensación económica por su participación. Se accedió a ellas a través de centros laborales y de asociaciones ciudadanas. Tras obtener el consentimiento informado, las pruebas fueron cumplimentadas de forma individual, o bien autocumplimentadas o bien, en forma de entrevista estructurada llevada a cabo por estudiantes de psicología previamente formados para ello, lo cual se hacía en función del nivel cultural. El presente estudio se inserta dentro de una investigación más amplia sobre las diferencias de género en salud. 


\section{Resultados}

En el MANOVA en que las variables dependientes fueron la sintomatología y los factores, el género (hombre, mujer) y el estatus menopáusico-edad (edad entre 20 y 31 años, premenopausia-edad entre 41 y 51 años, perimenopausia- edad entre 44 y 56 años y postmenopausia-edad entre 52 y 65 años, la interacción género $\mathrm{X}$ estatus menopáusicoedad fue estadísticamente significativa, $F$ (15, $3260)=3.85, p<0.001$, por lo que se realizaron, en primer lugar, análisis de varianza de forma independiente para cada grupo de edad/ estatus menopaúsico y, posteriormente, para cada género. En todos ellos se consideró la sintomatología como variable dependiente.

En el MANOVA realizado en el grupo de edad entre 20 y 31 años en que el factor fue el género se encontraron diferencias estadísticamente significativas, $F(5,304)=$ 2.30, $p<0.05$. Como puede observarse en la Tabla 2, los ANOVAs evidenciaron que solo había diferencias estadísticamente significativas en hipertensión, que era mayor en los hombres que en las mujeres. En el MANOVA realizado en el grupo de premenopausia-edad entre 41 y 51 años también se encontraron diferencias estadísticamente significativas, $F(5,458)=$ 5.74, $p<0.001$. Los ANOVAs mostraron que las mujeres puntuaron significativamente más alto que los hombres en síntomas vasomotores, mientras que estos puntuaban más alto que las mujeres en hipertensión. También se encontraron diferencias estadísticamente significativas en el MANOVA realizado en el grupo de perimenopausia-edad entre 44 y 56 años, $F(5,159)=13.40, p<$ 0.001. Los ANOVAs mostraron diferencias estadísticamente significativas en 3 de los 5 síntomas, puntuando las mujeres más que los hombres en síntomas vasomotores, palpitaciones e insomnio. También se encontraron diferencias estadísticamente significativas en el MANOVA realizado en el grupo de postmenopausia-edad entre 52 y 65 años, $F(, 248)=4.91, p<0.001$. Los ANOVAs mostraron que las mujeres tenían significativamente más síntomas vasomotores, más algias y más insomnio que los hombres.

\section{TABLA 2}

Medias, desviaciones típicas y análisis de varianza en sintomatología entre mujeres y hombres de diferente estatus menopáusico y/o edad

\begin{tabular}{|c|c|c|c|c|c|c|c|}
\hline \multirow{3}{*}{ Grupo } & \multicolumn{3}{|c|}{ MUJERES } & \multicolumn{2}{|c|}{ HOMBRES } & \multirow{2}{*}{\multicolumn{2}{|c|}{$\eta^{2}$}} \\
\hline & Sintomas & $M$ & $D T$ & $M$ & $D T$ & & \\
\hline & & & & & & $F$ & parcial \\
\hline \multirow{5}{*}{ 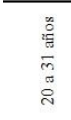 } & Vasomotores & 1.39 & 1.71 & 1.26 & 1.68 & 0.45 & \\
\hline & Algias & 5.98 & 3.82 & 6.06 & 3.98 & 0.03 & \\
\hline & Hipertensión & 0.28 & 0.68 & 0.54 & 1.01 & $7.33 * *$ & 0.023 \\
\hline & Palpitaciones & 0.69 & 0.99 & 0.63 & 1.03 & 0.23 & \\
\hline & Insomnio & 0.83 & 1.10 & 0.70 & 1.05 & 1.24 & \\
\hline \multirow{4}{*}{ 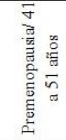 } & Vasomotores & 1.30 & 1.58 & 0.97 & 1.49 & $5.36^{*}$ & 0.011 \\
\hline & Algias & 6.79 & 4.08 & 6.17 & 4.01 & 2.62 & \\
\hline & Hipertensión & 0.38 & 0.83 & 0.69 & 1.03 & $13.4^{* * *}$ & 0.028 \\
\hline & Palpitaciones & $\begin{array}{l}0.63 \\
0.87\end{array}$ & $\begin{array}{l}1.01 \\
1.13\end{array}$ & $\begin{array}{l}0.65 \\
0.89\end{array}$ & 1.02 & $\begin{array}{l}0.05 \\
0.06\end{array}$ & \\
\hline \multirow{5}{*}{ 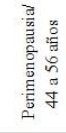 } & $\begin{array}{l}\text { Insomnio } \\
\text { Vasomotores }\end{array}$ & 2.81 & 1.92 & 0.94 & 1.48 & $56.2 * * *$ & 0.257 \\
\hline & Algias & 7.32 & 4.22 & 6.19 & 4.10 & 3.01 & \\
\hline & Hipertensión & 0.79 & 1.10 & 0.78 & 1.04 & 0.01 & \\
\hline & Palpitaciones & 1.19 & 1.24 & 0.55 & 0.93 & $14.1 * * *$ & 0.080 \\
\hline & Insomnio & 1.41 & 1.19 & 0.85 & 1.15 & $9.46^{* *}$ & 0.055 \\
\hline \multirow{5}{*}{ 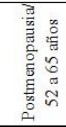 } & Vasomotores & 1.76 & 1.68 & 1.22 & 1.67 & $6.67^{*}$ & 0.026 \\
\hline & Algias & 7.28 & 4.25 & 6.08 & 4.01 & $5.34 *$ & 0.021 \\
\hline & Hipertensión & 0.78 & 1.04 & 0.96 & 1.09 & 1.76 & \\
\hline & Palpitaciones & 0.74 & 1.04 & 0.69 & 0.97 & 0.20 & \\
\hline & Insomnio & 1.36 & 1.14 & 0.86 & 1.19 & $12.1 * *$ & 0.046 \\
\hline
\end{tabular}

Notas: $* p<0.05 ; * *_{p}<0.01 ; * * p<0.001$.

Fuente: elaboración propia.

El MANOVA realizado en la muestra de hombres en los que se consideró como factor el grupo de edad, y como variable dependiente la sintomatología, evidenció la existencia de diferencias solo marginalmente significativas desde el punto de vista estadístico, $F(15,1563)=1.59, p=$ 0.07. Los ANOVAs mostraron diferencias estadísticamente significativas únicamente en hipertensión, $F(3,570)=3.79, p<0.05$. Los análisis post hoc con ajuste de Bonferroni para ver entre qué grupos se daban tales diferencias mostraron que eran estadísticamente significativas $(p<0.01)$ únicamente entre los hombres entre 20 y 31 años y los que tenían entre 52 y 65 , siendo más común la hipertensión en este último grupo.

El MANOVA realizado en la muestra de mujeres en el que se consideró como factor el estatus menopáusico y/o la edad mostró la existencia de diferencias estadísticamente significativas, $F(15,1687)=7.66, p<$ 0.001. Los ANOVAs evidenciaron que se daban diferencias estadísticamente significativas en síntomas vasomotores, $F(3,615)=20.98, p$ 
$<0.001$; algias, $F(3,615)=3.10, p<0.05$; hipertensión, $F(3,615)=12.16, p<0.001$, palpitaciones, $F(3,615)=6.28, \mathrm{p}<0.001 ; \mathrm{y}$ en insomnio, $F(3,615)=10.25, p<0.001$. Los análisis post hoc con ajuste de Bonferroni en síntomas vasomotores mostraron que se daban diferencias estadísticamente significativas entre las mujeres en perimenopausia y el resto de mujeres, las cuales tenían menor sintomatología (véase Figura 1).

\section{Figura 1}

Sintomas vasomotores en mujeres y hombres

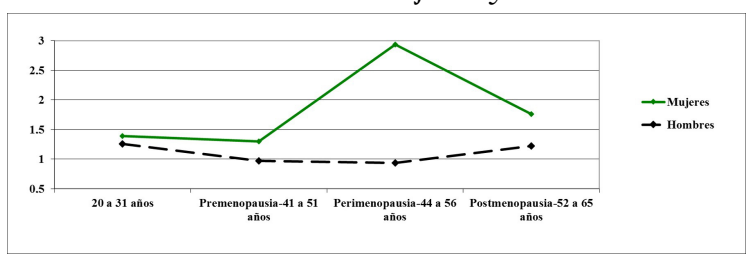

Fuente: elaboración propia.

Los análisis post hoc con ajuste de Bonferroni realizados para conocer entre qué grupos de mujeres se daban diferencias estadísticamente significativas en algias mostraron que se daban entre las de 52 y 65 años y las que tenían entre 20 y 31 años, las cuales informaban de menor dolor, tal y como ilustra la Figura 2.

\section{Figura 2}

Algias en mujeres y hombres

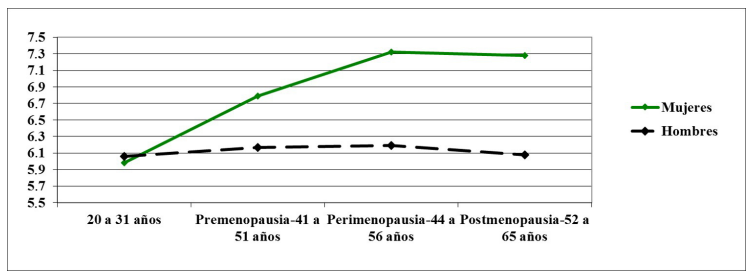

Fuente: elaboración propia.

Los análisis post hoc con ajuste de Bonferroni realizados para comprobar entre qué grupos de mujeres se daban diferencias estadísticamente significativas en hipertensión mostró que se daban entre las de edades entre 20 y 31 años y las que estaban en premenopausia y sus edades estaban entre 41 y 51 años, con respecto a las que se encontraban en perimenopausia y las que estaban en postmenopausia. Tal y como se ilustra en la Figura 3, las mujeres de menor edad son quienes informan con menor frecuencia de hipertensión.

Figura 3

Hipertensión de mujeres y hombres

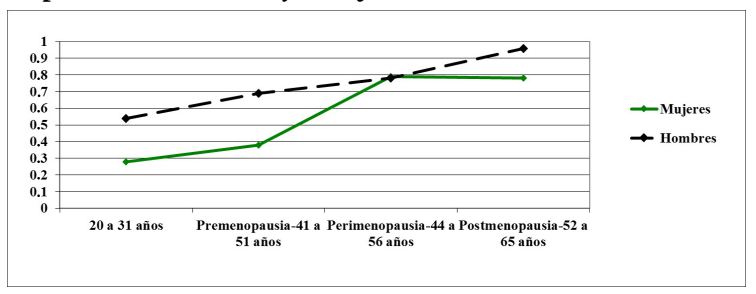

Fuente: elaboración propia.

Los análisis post hoc con ajuste de Bonferroni realizados para conocer entre qué grupos de mujeres se daban diferencias estadísticamente significativas en palpitaciones mostraron que ello sucedía entre las mujeres en perimenopausia y los otros tres grupos de mujeres, informando de más palpitaciones las mujeres en dicha fase (véase Figura 4).

\section{Figura 4}

Palpitaciones en mujeres y hombres

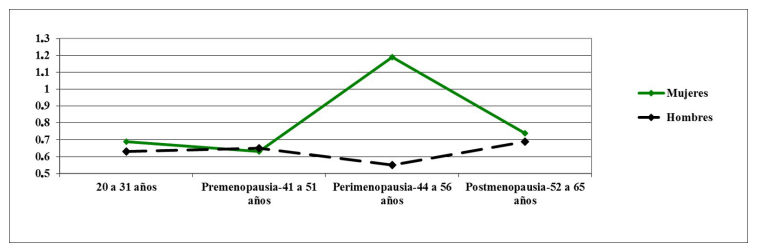

Fuente: elaboración propia.

Los análisis post hoc con ajuste de Bonferroni realizados para conocer entre qué grupos de mujeres se daban diferencias estadísticamente significativas en insomnio, mostraron que se daban entre los dos grupos de menor edad respecto a las que estaban en perimenopausia y las que estaban en postmenopausia, informando las mujeres de estos dos últimos grupos de más insomnio, tal y como se muestra en la Figura 5. 
Figura 5

Insomnio de mujeres y hombres

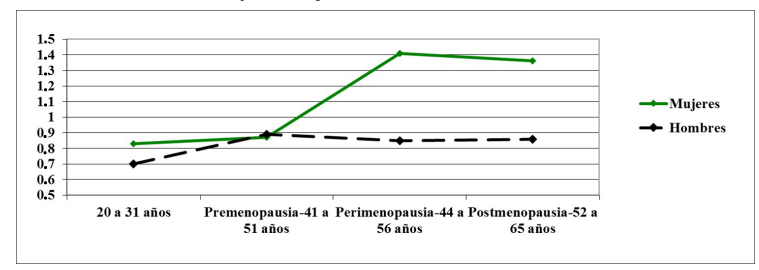

Fuente: elaboración propia.

Para conocer la asociación entre el apoyo social percibido en los diferentes grupos de edad y/o estatus menopáusico y los problemas de salud estudiados se hicieron correlaciones utilizando el coeficiente de correlación de Pearson, resultados que se muestran en la Tabla 3. Como puede observarse, se dan algunas asociaciones estadísticas significativas entre apoyo social y salud, si bien tal asociación depende del indicador de salud considerado, del género y del estatus menopáusico y/o de la edad. Aunque el porcentaje de varianza común es bajo, se encontró de forma consistente y en ambos géneros que el apoyo social se asociaba con menos insomnio, si bien ello no se detectó en las mujeres en perimenopausia. Además, también se encontró una cierta tendencia a informar de menos palpitaciones en las mujeres y los hombres con mayor apoyo social. El mayor efecto protector del apoyo social se dio en las mujeres en premenopausia y postmenopausia, tal y como se observa en la Tabla 3.

\section{TABLA 3}

Coeficientes de correlación de Pearson entre sintomatología y apoyo social emocional e instrumental en mujeres y hombres de cada grupo de edad/estatus menopáusico

\begin{tabular}{|c|c|c|c|c|c|}
\hline \multirow[b]{2}{*}{ Grupo } & \multicolumn{3}{|c|}{ MUJERES } & \multicolumn{2}{|c|}{ HOMBRES } \\
\hline & Sintomas & Emocional & Instrumental & Emocional & Instrumental \\
\hline \multirow{5}{*}{ 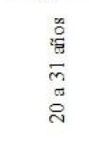 } & Vasomotores & 0.10 & 0.05 & -0.080 & 0.04 \\
\hline & Algias & -0.04 & -0.08 & -0.15 & $-0.25 * *$ \\
\hline & Hipertensión & $-0.16^{*}$ & -0.10 & -0.15 & -0.02 \\
\hline & Palpitaciones & -0.13 & -0.15 & $-0.22 * *$ & -0.09 \\
\hline & Insomnio & $-21 * *$ & $-0.26^{* *}$ & $-0.23^{* *}$ & $-0.20 *$ \\
\hline \multirow{5}{*}{ 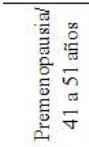 } & Vasomotores & $-0.20 *$ & $-0.15^{* *}$ & -0.20 ** & -0.11 \\
\hline & Algias & $-0.29 * * *$ & $-0.13^{*}$ & -0.11 & -0.09 \\
\hline & Hipertensión & $-0.016 * *$ & $-0.13 * *$ & -0.08 & -0.01 \\
\hline & Palpitaciones & $-0.29 * * *$ & $-0.19 * *$ & $-0.17^{*}$ & -0.06 \\
\hline & Insommio & $-18 * *$ & -0.08 & $-0.19 * *$ & $-0.18^{*}$ \\
\hline \multirow{5}{*}{ 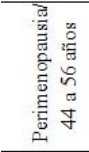 } & Vasomotores & -0.20 & -0.20 & 0.06 & 0.07 \\
\hline & Algias & -0.15 & -0.08 & 0.02 & 0.06 \\
\hline & Hipertensión & -0.06 & -0.10 & 0.00 & 0.02 \\
\hline & Palpitaciones & -0.16 & -0.21 & -0.19 & $0.24^{*}$ \\
\hline & Insomnio & -13 & -0.10 & -0.06 & -0.14 \\
\hline \multirow{5}{*}{ 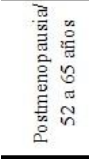 } & Vasomotores & -0.16 & $-0.22 * *$ & -0.09 & -0.11 \\
\hline & Algias & $-0.34 * * *$ & $-0.39 * * *$ & -0.09 & -0.11 \\
\hline & Hipertensión & $-0.26 * *$ & $-0.30 * *$ & -0.15 & -0.13 \\
\hline & Palpitaciones & $-0.41 * * *$ & $-0.36 * * *$ & $-0.19 *$ & -0.15 \\
\hline & Insomnio & $-24 * *$ & $-0.25 * *$ & $-0.24 * *$ & $-0.22 *$ \\
\hline
\end{tabular}

\section{Discusión}

Los resultados del presente estudio evidencian que, aunque las mujeres no informan de peor salud que los hombres cuando son jóvenes, periodo en el que más bien sucede el fenómeno opuesto, a partir de los 40 años informan de más sintomatología que ellos. Y, aunque el estatus menopáusico parece ser un factor importante de algunas de las diferencias encontradas, la evidencia no es completa ya que la existencia de más síntomas vasomotores en mujeres que en hombres se comienza a detectar desde la premenopausia. Además, cuando se encontraron diferencias estadísticamente significativas, el tamaño del efecto de las diferencias entre mujeres y hombres fue bajo, excepto en la sintomatología vasomotora durante la perimenopausia. También en otros países se ha encontrado que las diferencias en salud entre mujeres y hombres difieren en función del síntoma y del grupo de edad que se considere, de forma que no existieron diferencias en función del género en algunos indicadores de salud (Walters et al., 2002). 
Pese a ello, destaca que la interacción género por estatus menopáusico-edad fue estadísticamente significativa en los síntomas de tipo vasomotor, en palpitaciones y en insomnio lo que sugiere que tales indicadores de salud evolucionan de forma diferente en mujeres y hombres a lo largo del periodo adulto. De hecho, excepto en hipertensión, que era más común en los hombres de mayor edad respecto a los más jóvenes, no se detectaron diferencias significativas en función del grupo de edad en los hombres; pero sí hubo diferencias estadísticamente significativas entre las mujeres con diferente edad y/o estatus menopáusico, las cuales tenían peor salud durante la perimenopausia, resultados que coinciden con los encontrados en otros estudios (Avis et al., 2001). Sin embargo, mientras que la sintomatología de tipo vasomotor y las palpitaciones parecen ser bastante específicas de este periodo, siendo menores en las mujeres que ya han superado esta fase y antes de ella, esto no sucede con el insomnio y la hipertensión, que se mantienen durante la postmenopausia. También en otros estudios se ha evidenciado que durante la premenopausia las mujeres tienen menos sintomatología que en la perimenopausia y la postmenopausia (Pimenta et al., 2012).

Como ya se ha citado, se encontró que a partir de los 40 años las mujeres informan de más síntomas vasomotores que los hombres, si bien el tamaño del efecto es mucho mayor en la perimenopausia. Aunque tales síntomas no son independientes de los factores culturales y de estilo de vida (cfrs. Dhanoya et al., 2016; Mansikkamäki et al., 2016), son los que se han asociado de forma más consistente con la menopausia (Avis et al., 2005). También durante este periodo las mujeres informan de más insomnio y palpitaciones que los hombres y, aunque esta última diferencia no se detectó durante la postmenopausia, en esta última fase las mujeres informan también de más dolores de cabeza y osteomusculares que los hombres, los cuáles son también significativamente más comunes que en las mujeres más jóvenes.

Los análisis de la asociación entre el apoyo social percibido y la salud han evidenciado que el apoyo social tiene un cierto papel protector de la salud, sobre todo en las mujeres en postmenopausia. En los hombres, el efecto protector del apoyo social parece limitarse al insomnio y las palpitaciones. También en otros estudios se ha encontrado que el apoyo social percibido, sobre todo el emocional, se asocia con menor patología cardiovascular, y se relaciona en las mujeres, pero no en los hombres, con menor tensión arterial (Bowen et al., 2013).

Aunque el presente estudio tiene limitaciones tales como el tratarse de un estudio transversal y que toda la información se ha obtenido mediante autoinforme, los resultados evidencian que la menopausia es una fase en que las mujeres presentan mayor sintomatología que los hombres, pero en la salud de las mujeres también parece importante el apoyo social, sobre todo a partir de la menopausia y antes de esta. Los resultados del presente estudio pueden ser de utilidad para el diseño de estrategias y programas que ayuden a desterrar los mitos en torno a la menopausia y promuevan un envejecimiento saludable en las mujeres.

\section{Agradecimientos}

Investigación subvencionada por el Ministerio de Economía y Competitividad. Referencia: FEM2012-34632

\section{Referencias}

Avis, N. E., Brockwell, S., \& Colvin, A. (2005). A universal menopausal syndrome? The American Journal of Medicine, 118(12), 37S-47S. http://dx.doi.org/10.1016/j.amjm ed.2005.09.057

Avis, N. E., Stellato, R., Crawford, S., Bromberger, J., Ganz, P., Cain, V., \& Kagawa-Singer, M. (2001). Is there a menopausal syndrome? Menopausal status and symptoms across racial/ethnic groups. Social Science and Medicine, 52 (3), 345-356. Recuperado de https://www.ncbi.nlm.nih.g ov/pubmed/11330770 
Bowen, K. S., Birmingham, W., Uchino, B. N., Carlisle, M., Smith, T. W., \& Light, K. C. (2013). Specific dimensions of perceived support and ambulatory blood pressure: Which support functions appear most beneficial and for whom? International Journal of Psychophisiology, 88(3), 317-324. http://dx.doi.org/10.1016/j .ijpsycho.2012.03.004

Calvaresi, E., \& Bryan, J. (2003). Symptom experience in Australian men and women in midlife. Maturitas, 44(3), 225-236. http://dx.doi.org/10.1016/S03785122(03)00037-9

Cuadros, J. L., Fernández-Alonso, A. M., Cuadros-Celorrio, A. M., FernándezLuzón, N., Guadix-Peinado, M. J., CidMartín, N.,... Pérez-López, F. R. (2012). Perceived stress, insomnia and related factors in women around the menopause. Maturitas, 72(4), 367-372. http://dx.doi.org /10.1016/j.maturitas.2012.05.012

Dhanoya, T., Sievert, L. L., Muttukrishna, S., Begum, K., Sharmeen, T., Kasim, A., ... Bentley, G. R. (2016). Hot flushes and reproductive hormone levels during the menopausal transition. Maturitas, 89, 43-51. http://dx.doi.org/10.1016/ j.maturitas.2016.03.017

Duffy, O. K., Iversen, L., \& Hannaford, P. C. (2013). Factors associated with reporting classic menopausal symptoms differ. Climateric, 16(2), 240-251. http://dx. doi.org/10.3109/13697137.2012.697227

Extremera, N., Rey, L., \& Pena, M. (2010). La docencia perjudica seriamente la salud. Boletín de Psicología, 100, 43-54. Recuperado de http://www.uv.es/seoane/bo letin/previos/N100-3.pdf

Emslie, C., Hunt, K., \& Macintyre, S. (1999). Gender differences in minor morbidity among full time employees of a British university. Journal of Epidemiology Eु Community Health, 53(8), 465-475. Recuperado de https://www.ncbi.nlm.nih.g ov/pmc/articles/PMC1756947/

García-Astudillo, E., Pinto-García, M. P., \& Laguna-Sáez, J (2015). Incontinencia urinaria: frecuencia y factores asociados. Fisioterapia, 37(4), 145-154. http://dx.doi.o $\mathrm{rg} / 10.1016 / \mathrm{j} . \mathrm{ft} .2014 .09 .002$

Herrera, V., \& Guarino, L. (2008). Sensibilidad emocional, estrés y salud percibida en cadetes navales venezolanos. Universitas Psychologica, 7(2), 185-198. Recuperado de http://revistas.javeriana.edu.co/index.p hp/revPsycho/article/viewFile/208/225

Kroenke, K., \& Spitzer, R. L. (1998). Gender differences in reporting of physical and somatoform symptoms. Psychosomatic Medicine, 60(2), 150-155. Recuperado de https://www.ncbi.nlm.nih.gov/pubmed/ 9560862

Mansikkamäki, K., Nygårdc, C., Raitanena, J., Kukkonen-Harjulaa, K., Tomás, E. Rutanenc, R., \& Luoto, R. (2016). Hot flushes among aging women: A 4-year follow-up study to a randomised controlled exercise trial. Maturitas, 88, 84-89. http://d x.doi.org/10.1016/j.maturitas.2016.03.010

Marcus-Newhall, A., Thompson, S., \& Thomas, C. (2001). Examining a gender stereotype: menopausal women. Journal of Applied Social Psychology, 31(4), 698-719. http://dx.doi.org/10.1111/j.15591816.2001.tb01409.x

Martínez-Garduño, M. D., González-Arratia, N. I., van Barneveld, H. O., \& DomínguezEspinosa, A. C. (2012). Satisfacción con la vida asociada al apoyo familiar en la perimenopausia y postmenopausia. Salud Mental, 35(2), 91-98.

Martínez-Pérez, J. A., Chavida, F., Palacios, S., \& Pérez, M. (2008). Epidemiology of risk factors and symptoms associated with menopause in Spanish women. Matutitas, 62 (1), 30-36. http://dx.doi.org/10.1016/j.m aturitas.2008.10.003

Matud, M. P. (1998). Investigación del estrés y su impacto en la salud de la mujer en Canarias. Memoria de investigación. Las Palmas de Gran Canaria: Instituto Canario de la Mujer.

Matud, M. P., Ibáñez, I., Bethencourt, J. M., Marrero, R., \& Carballeira, M. (2003). Structural gender differences in perceived 
social support. Personality and Individual Differences, 35(8), 1919-1929. http://dx.doi .org/10.1016/S0191-8869(03)00041-2

McDonough, P., \& Walters, V. (2001). Gender and health: Reassessing patterns and explanations. Social Science Eु Medicine, 52(4), 547-559. Recuperado de https://ww w.ncbi.nlm.nih.gov/pubmed/11206652

Meadows, M. (1989). Personality, stress, and health. (Tesis doctoral no publicada). Universidad de York, UK.

Melián, E. M. (2015). Útero, psiquis y climaterio: un acercamiento desde la endocrinología antropológica. Investigaciones Feministas, 6, 196-208. Recuperado de http://revistas.uc m.es/index.php/INFE/article/view/51500

Mendoza, N., Sánchez-Borrego, R., Cancelo, M. J., Calvo, A., Checa, M. A., Cortés, J., Elorriaga, M. A., ... De la Viuda, E. (2013). Position of the Spanish menopause society regarding the management of perimenopause. Maturitas, 74(3), 283-290. http://dx.doi.org/10.1016/j .maturitas.2012.12.010

Mishra, G. D., \& Kuh, D. (2012). Health symptoms during midlife in relation to menopausal transition: British prospective cohort study. British Medical Journal, 344, e402. https://doi.org/10.1136/bmj.e402

Moral-de la Rubia, J., Valdez-Medina, J. L., González-Arratia López-Fuentes, N. I., González-Escobar, S., Alvarado- Bravo, B. G., \& Gaona-Valle, L. S. (2010). Emociones y síntomas físicos medicamente no explicados en mujeres y hombres adultos. Medicina Universitaria, 12(47), 96-105.

Organización Mundial de la salud [OMS]. (1996). Research on the menopause in the 1990s. Geneva, Switzerland; World Health Organization. WHO Technical Report Series, 866. Recuperado de http://whqlibdo c.who.int/trs/WHO_TRS_866_spa.pdf

Organización Mundial de la Salud (1981). Investigaciones sobre la Menopausia. Ginebra: OMS, Serie de informes técnicos: 670. Recuperado de http://apps.who.int/iris/bitstream/10665 /41316/1/WHO_TRS_670_spa.pdf

Pimenta, F., Leal, I., Maroco, J., \& Ramos, C. (2012). Menopausal symptoms: Do life events predict severity of symptoms in peri- and post-menopause? Maturitas, 72 (4), 324-331. http://dx.doi.org/10.1016/j .maturitas.2012.04.006

Rincón, L. B., \& Guarino, L. R. (2008) estrés laboral, afrontamiento, sensibilidad Estrés laboral, afrontamiento, sensibilidad en médicos venezolanos. Revista Colombiana de Psicología, 17, 43-58. Recuperado de http://www.revistas.unal.edu.co/index.p hp/psicologia/article/view/1188

Romero-Pérez, I. M., Monterrosa-Castro, \& Paternina-Caicedo, A. (2010). Menopausia y etnias/razas: ihay diferencias en la representación de los síntomas? Revista Colombiana de Obstetricia y Ginecología, 61 (4), 319-328. Recuperado de http://www. scielo.org.co/pdf/rcog/v61n4/v61n4a05.pdf

Saiz-González, D., Rodríguez, M., García, C., Prieto, R., \& Saiz-Ruiz, J. (2009). Diferencias de sexo en el trastorno depresivo mayor: síntomas somáticos y calidad de vida. Revista de Psiquiatría y Salud Mental, 2(3), 119-127. http://dx.doi.org/10 .1016/S1888-9891(09)72402-4

Sánchez, O., \& Ledesma, R. D. (2009). Análisis Psicométrico del Inventario de Síntomas Revisado (scl-90-r) en Población Clínica. Revista Argentina de Clínica Psicológica, 18(3), 265-274.

Słopień R., Wichniak, A., Pawlak, M , Słopień, A., Warenik-Szymankiewicz, A., \& Sajdak, S. (2015). Disturbances of sleep continuity in women during the menopausal transition. Psychiatria Polska, 49(3), 615-623. http://dx.doi.org/10.12740 $/ \mathrm{PP} / 33442$

Spetz, A. C., Fredriksson, M. G., \& Hammar, M. L. (2003). Hot flushes in a male population aged 55,65 , and 75 years, living in the community of Linkoping, Sweden. Menopause, 10(1), 81-87. Recuperado de https://www.ncbi.nlm.nih.gov/pubmed/ 12544681 
Sturdee, D. W., \& Villiers, T. J. (2011). Menopause redefined. Climateric, 14(1), 3-4.

Van Hall, E., Verdel, M., \& van der Velden, J. (2009). "Perimenopausal" complaints in women and men: a comparative study. Journal of Women'Health, 3(1), 45-49. http://dx.doi.org/10.3109/13697137 .2011 .542102

Walters, V., McDonough, P., \& Strohschein, L. (2002). The influence of work, household structure, and social, personal and material resources on gender differences in health: an analysis of the 1994 Canadian National Population Health Survey. Social Science $\mathbb{E}$ Medicine, 54(5), 677-692. http://dx.doi.org/ 10.1016/S0277-9536(01)00117-4

Woods, N. F., \& Mitchell, E. S. (2005). Symptoms during the perimenopause: prevalence, severity, trajectory, and significance in women's lives. The American Journal of Medicine, 118(12), 14-24. http://dx.doi.org/ 10.1016/j.amjmed.2005.09.031

\section{Notas}

* Artículo de investigación. 